\title{
Differential expression of elastic fibre components in intrinsically aged skin
}

\author{
Abigail K. Langton • Michael J. Sherratt • \\ Christopher E. M. Griffiths • \\ Rachel E. B. Watson
}

Received: 11 February 2011/Accepted: 21 March 2011/Published online: 2 April 2011

(C) Springer Science+Business Media B.V. 2011

\begin{abstract}
Intrinsic ageing of the skin is a subtle process resulting in some degree of skin laxity. The dermal elastic fibre network imbues skin with the capacity to recoil and loss of this property contributes to an aged, wrinkled appearance. Whilst elastic fibres have a complex, composite structure which allows them to fulfil multiple roles, the effects of intrinsic ageing on their discrete molecular components has not previously been explored. In this study, we have used a microarray-based approach to perform a novel survey of the changes in gene expression that occur in components of cutaneous elastic fibres as a result of intrinsic ageing. Age-related changes in gene expression were validated at the mRNA and protein levels using quantitative real-time polymerase chain reaction (qPCR) and immunostaining, respectively. The microarray revealed that the majority of elastic fibre
\end{abstract}

Electronic supplementary material The online version of this article (doi:10.1007/s10522-011-9332-9) contains supplementary material, which is available to authorized users.

A. K. Langton $(\varangle)$ · C. E. M. Griffiths · R. E. B. Watson Dermatological Sciences, The University of Manchester, Manchester Academic Health Science Centre, 1.222

Stopford Building, Oxford Road, Manchester M13 9PT, UK

e-mail: abigail.langton@manchester.ac.uk

M. J. Sherratt

Regenerative Biomedicine, The University of Manchester, Manchester Academic Health Science Centre,

Manchester, UK network components were unchanged with age. However, three differentially expressed genes were identified: latent TGF $\beta$-binding protein (LTBP)-2 which was up-regulated with age (fold change +1.58 , $P=0.041$ ); LTBP3 (fold change $-1.67, P=0.025$ ) and the lysyl oxidase-like enzyme (LOXL1, fold change $-1.47, P=0.008$ ) which were both downregulated with age. Although the changes in gene expression for LTBP3 were not confirmed by either qPCR or immunostaining, the expression and tissue deposition of both LTBP2 and LOXL1 were significantly enhanced in intrinsically aged skin. Whilst the functional implications of these altered expression profiles remains to be elucidated, LTBP2 and LOXL1 are thought to play important roles in controlling and maintaining elastic fibre deposition, assembly and structure via binding to fibulin-5. Consequently, any age-related perturbations in the expression of these components may have important consequences on remodelling of the extracellular matrix and hence on the mechanical properties of intrinsically aged skin.

Keywords Elastic fibre - Intrinsic ageing · Fibrillin-rich microfibril
Abbreviations
AUC Area under the curve
bp Base pair
DAPI 4'-6-Diamidino-2-phenylindole
DEJ Dermal-epidermal junction
GC-RMA GeneChip-Robust Multiarray Averaging 


$\begin{array}{ll}\text { LOX } & \text { Lysyl oxidase } \\ \text { LOXL } & \text { Lysyl oxidase-like } \\ \text { LTBP } & \text { Latent transforming growth factor- } \beta \\ & \text { binding protein } \\ \text { qPCR } & \text { Quantitative real-time polymerase chain } \\ & \text { reaction } \\ \text { TBS } & \text { Tris-buffered saline }\end{array}$

\section{Introduction}

Whilst facial wrinkles occur primarily as a consequence of chronic exposure to environmental insults such as sunlight (Fisher et al. 1996) and smoking (Ernster et al. 1995; Kadunce et al. 1991), in the absence of such exogeneous factors, the clinical effects of chronological (also known as intrinsic ageing) may only become apparent from the age of 70 years (Kafi et al. 2007). This slow ageing process causes subtle changes in tissue structure (Montagna and Carlisle 1979) which in turn impairs function. As a consequence, intrinsically aged skin is relatively smooth, finely wrinkled and unblemished. Histologically, whilst few changes are noted in the epidermis, the topology of the dermal-epidermal junction (DEJ) is altered in that it exhibits a flattened appearance with a correspondingly diminished connecting surface area (Giangreco et al. 2010). Intrinsically aged skin is also more fragile and less elastic (both resilience and compliance) than young skin (Escoffier et al. 1989).

The dermal elastic fibre network, which is a major contributor to elastic recoil (Uitto 1979), is composed of complex and highly ordered macromolecular assemblies (Cotta-Pereira et al. 1976). Discrete cascades of candelabra-like oxytalan fibres (which are composed solely of fibrillin-rich microfibrils) connect the DEJ to fibrillin and elastin containing elaunin fibres in the lower papillary dermis. Finally, in the reticular dermis, large diameter elastin-rich fibres run parallel to the DEJ (for a review see (Langton et al. 2010). Hence, with increasing depth from the DEJ, the ratio of elastin to fibrillin increases. The developmental deposition of elastic fibres is also highly regulated: tropoelastin, the soluble precursor of elastin, is deposited onto a pre-formed fibrillin-rich microfibrillar scaffold. Mature elastin is then formed via the enzyme-mediated cross-linking of tropoelastin by lysyl oxidase (LOX) and/or LOX-like (LOXL) proteins (Mithieux and Weiss 2005). In the mature elastic fibre, the elastin core comprises over $90 \%$ of the volume whilst the fibrillin-rich microfibrils are largely confined to an outer mantle (Mecham and Davis 1994). Although elastin and fibrillin-1 are the predominant protein constituents of skin-derived elastic fibres, several proteins, including the microfibril associated glycoproteins (MAGPs), latent transforming growth factor- $\beta$ binding proteins (LTBPs) and fibulins are frequently associated with microfibrils, whilst decorin, biglycan, versican and the emilins may also be sporadically co-localised (Kielty et al. 2002).

Both the intrinsic and extrinsic ageing processes have profound, yet differing effects on the structure, and hence potentially the function, of the elastic fibre network (Sherratt 2009). The characteristic smooth appearance and fine wrinkles of intrinsically aged skin are associated with a progressive fragmentation and decline of the elastic fibre network (Braverman and Fonferko 1982; Montagna and Carlisle 1979). In contrast, the deep wrinkles that characterise photoaged skin are associated with not only loss of the fibrillin-rich microfibrils at the DEJ (Watson et al. 1999) but crucially the gain of elastotic material (disorganised elastic fibre proteins) within the deep dermis (Watson et al. 2001). These architectural and compositional alterations are thought to occur as a result of structural degenerative changes within the elastic fibre which may be mediated by cellular mechanisms, principally extracellular proteases (Kielty et al. 1994; Ashworth et al. 1999) and/or by cellular mechanisms such as exposure to ultraviolet radiation (Sherratt et al. 2010).

Although these elastic fibre components are required to fulfil important yet disparate mechanical and biochemical roles in structurally and functionally dissimilar ocular, vascular and cutaneous tissues, the effects of intrinsic ageing on this group of biomolecules has not previously been explored. Therefore, the aim of this study was to perform a novel survey of the changes that occur in commonly-associated elastic fibre components in young and intrinsically aged human skin. Age-related changes in cutaneous gene expression were identified using an Affymetrix ${ }^{\circledR}$ microarray and were validated at the mRNA and protein levels using quantitative real-time polymerase chain reaction (qPCR) and immunostaining, respectively. 


\section{Materials and methods}

Skin biopsy procurement

Skin biopsies (6 $\mathrm{mm}$ diameter) were obtained from the photoprotected buttock of nine young (five men and four women; age range 18-29 years) and nine aged (five men and four women; age range 65-75 years) healthy Caucasian volunteers. Each punch biopsy was obtained under $1 \%$ lignocaine local anaesthesia and bisected. One half of the biopsy was immersed in RNAlater solution (Ambion, Foster City, CA, USA) and stored at $-80^{\circ} \mathrm{C}$, the remaining half was embedded in optimal cutting temperature compound (Miles Laboratories, Elkhart, IN, USA), snap frozen in liquid nitrogen and stored at $-80^{\circ} \mathrm{C}$. Local ethical committee approval was obtained for the study and all subjects gave written, informed consent.

\section{RNA extraction}

Total RNA was isolated from frozen biopsy tissue using reagents from the TRIzol ${ }^{\circledR}$ Plus RNA Purification Kit (Invitrogen, Paisley, UK) according to manufacturer's instructions. Traces of genomic DNA were minimised by DNase I treatment (PureLink $^{\mathrm{TM}}$ DNase, Invitrogen) of the samples. The concentration and purity of RNA for each sample was determined by UV absorbance using a Nanodrop ND1000 spectrophotometer (Fisher Scientific, Loughborough, UK).

\section{Microarray analysis}

Microarray experiments were performed using the human genome U133A oligonucleotide array (Affymetrix Inc., High Wycombe, UK) according to the manufacturer's instructions. Total RNA (100 ng) from three young (age range 24-26 years) and three aged (age range 74-75 years) male volunteers was used with the Two-Cycle cDNA Synthesis Kit (P/N 900432 Affymetrix Inc.; one sample hybridized per array). Technical quality control was performed with dChip (Li and Wong 2001). Background correction, quantile normalization and gene expression analysis were performed using GeneChip-Robust Multiarray Averaging (GC-RMA) (Wu et al. 2004). Differential expression between the young and aged groups was tested statistically with CyberT on logarithmic scale data (Baldi and Long 2001). False-discovery correction was performed with Q-value software (Storey and Tibshirani 2003). Significantly changed probe sets were selected on $P<0.05$ and expression level $(>50)$.

\section{Real-time qPCR}

cDNA was transcribed from total RNA using random primers and the cloned AMV first-strand cDNA synthesis kit (Invitrogen). The resulting cDNA was subjected to qPCR analysis using human TaqMan ${ }^{\circledR}$ gene expression assays (Applied Biosystems, Warrington, UK) and the StepOne Plus ${ }^{\mathrm{TM}}$ Real-Time PCR system (Applied Biosystems). TaqMan ${ }^{\circledR}$ gene expression assays were obtained for LTBP2 (Hs01553358_m1, RefSeq: NM_000428.2, amplicon size $=64$ bp), LTBP3 (Hs01105729_m1, RefSeq: NM_001130144.2, amplicon size $=86 \mathrm{bp}$ ) and LOXL1 (Hs00935937_m1, RefSeq: NM_005576.2, amplicon size $=73 \mathrm{bp}$ ). Two types of controls were included for each gene analysed: (i) reverse transcription mixture synthesised without reverse transcriptase, aimed at detecting genomic DNA contamination in the RNA sample and (ii) a no cDNA template well was used to test for contamination of assay reagents. For each gene analysed, all samples were run on the same 96-well plate. Each reaction well contained a total volume of $20 \mu \mathrm{l}$, consisting of: $10 \mu \mathrm{l}$ of $\operatorname{TaqMan}^{\circledR}$ Universal PCR Master Mix (Applied Biosystems), $1 \mu \mathrm{l} 20 \times$ Taq$\mathrm{Man}^{\circledR}$ gene expression assay, $8 \mu \mathrm{l}$ RNase-free water and $1 \mu \mathrm{l}$ cDNA template. The qPCR conditions were as follows: denaturation at $95^{\circ} \mathrm{C}$ for $20 \mathrm{~s}$, followed by 40 cycles of amplification and quantification $\left(95^{\circ} \mathrm{C}\right.$ for $1 \mathrm{~s}$ and $60^{\circ} \mathrm{C}$ for $20 \mathrm{~s}$ ). Expression ratios were determined relative to a standard sample and normalized using the value derived from the housekeeping gene ACTB (TaqMan $^{\circledR}$ Human ACTB Endogenous Control, RefSeq: NM_001101.2, amplicon size $=171 \mathrm{bp}, \quad$ Applied Biosystems). Fold changes were calculated relative to young skin and represent data from triplicate experiments. Data were analysed by Student's $t$-test (calculated using SPSS + v16.0 software, SPSS Inc., Chicago, IL, U.S.A.) and results were considered significant if $P<0.05$ (95\% confidence level). 
Immunofluorescence staining

Frozen tissue sections $(5 \mu \mathrm{m})$ were fixed in ice-cold acetone and hydrated in tris-buffered saline (TBS, $100 \mathrm{mmol} \mathrm{l}^{-1}$ Tris, $150 \mathrm{mmol} \mathrm{l}^{-1} \mathrm{NaCl}$; pH 7.4). Non-specific binding was blocked by incubation in $5 \%$ normal serum. Primary antibodies were applied for $1 \mathrm{~h}$ at room temperature. These were rabbit antihuman LTBP2 primary antibody (Sigma Prestige Antibodies $^{\mathrm{TM}}$, Poole, UK) diluted 1:100, rabbit antihuman LTBP3 primary antibody (Santa Cruz Biotechnology Inc., Santa Cruz, CA, USA, clone H-210) dilution 1:10, mouse anti-human LOXL1 primary antibody (Santa Cruz Biotechnology Inc., clone H-11) dilution 1:10. Primary antibodies were omitted from selected sections as a negative control. Sections were washed in TBS, prior to incubation in the appropriate fluorescent secondary antibody (Alexa Fluor $^{\circledR}$, Invitrogen). Nuclei were visualised by incubation of sections in 4'-6-Diamidino-2-phenylindole (DAPI).

\section{Immunohistochemical staining}

Frozen sections $(5 \mu \mathrm{m})$ were fixed in $4 \%$ paraformaldehyde and hydrated in TBS. Sections were pretreated with $0.5 \%$ Triton $\mathrm{X}-100$ and endogenous peroxidase activity abolished by incubation with $3 \%$ hydrogen peroxide in methanol. Non-specific binding of the secondary antibody was blocked by incubation with 3\% normal serum. Mouse anti-human LTBP2 primary antibody (Sigma, clone 5D7, diluted 1:100) was applied overnight at $4^{\circ} \mathrm{C}$. Primary antibody was omitted from selected sections as a negative control. Sections were washed in TBS, prior to incubation with the appropriate biotinylated secondary antibody for $30 \mathrm{~min}$. Antibody staining was visualized using a well-characterized immunoperoxidase reaction (VectaStain Elite $\mathrm{ABC}$ system; Vector Laboratories, Burlingame, CA, U.S.A.) utilising Vector $\mathrm{SG}^{\circledR}$ as chromogen. Following light counterstaining with nuclear fast red, sections were dehydrated and permanently mounted.

Image capture and analysis

Images were captured using the All-in-one Type Fluorescence Microscope Biozero-8000 (Keyence, Osaka, Japan). The degree of immunostaining was measured using ImageJ software (Abramoff et al. 2004) and was assessed for significance using Student's $t$-test. Results were considered significant if $P<0.05$ (95\% confidence level).

\section{Results}

Intrinsic skin ageing induced subtle changes in the gene expression levels of elastic fibre components

Putative elastic fibre network components were identified from the literature (Kielty et al. 2002) and a list of gene expression fold change for these components was compiled (Table 1 and Supplementary Table 1). In total, 29 components of the elastic fibre network were examined and of these, 26 showed no significant change in gene expression with age. Seven genes were found to be below the level of detection (expression level <50) for all probe sets represented. However, three differentially expressed probe sets were identified for components of elastic fibres (Table 1 and Fig. 1). LTBP2 was significantly up-regulated with age (fold change: +1.58 , $P=0.041)$ whilst LTBP3 and the LOXL1 were significantly down-regulated (LTBP3 fold change $-1.67, P=0.025$; LOXL1 fold change -1.47 , $P=0.008)$. In order to validate the microarray data, qPCR was performed using human TaqMan ${ }^{\circledR}$ gene expression assays for these differentially expressed genes. We also employed immunological approaches to establish whether age-related changes in gene expression were correlated with changes in protein abundance and distribution within these tissues. These validation investigations were performed on the same young and aged biopsy samples used to generate the microarray data and on additional biopsy samples from photoprotected buttock (seven young volunteer samples: three men and four women, age range 18-29 years; and seven aged volunteer samples: three men and four women, age range 65-75 years).

LTBP3 expression and tissue distribution is not significantly altered with advancing age

Whilst the initial microarray data suggested that LTBP3 expression was down-regulated with age, 
Table 1 Age-related changes in gene expression for commonly-associated elastic fibre components

\begin{tabular}{|c|c|c|c|c|}
\hline Affymetrix ${ }^{\circledR}$ ID & $\mathrm{Gene}^{\mathrm{a}}$ & Gene (description) & $P$-value & $\mathrm{FC}^{\mathrm{b}}$ \\
\hline 219922_s_at & LTBP3 & Latent transforming growth factor $\beta$ binding protein 3 & 0.025 & -1.67 \\
\hline 203570_at & LOXL1 & Lysyl oxidase-like 1 & 0.008 & -1.47 \\
\hline 203417_at & MAGP1 & Microfibril-associated glycoprotein 1 & 0.082 & -1.35 \\
\hline 212713_at & MFAP4 & Microfibrillar-associated protein 4 & 0.629 & -1.20 \\
\hline 213428_s_at & COL6A1 & Collagen, type VI, alpha 1 & 0.289 & -1.20 \\
\hline 202729_s_at & LTBP1 & Latent transforming growth factor $\beta$ binding protein 1 & 0.501 & -1.15 \\
\hline 204442_x_at & LTBP4 & Latent transforming growth factor $\beta$ binding protein 4 & 0.304 & -1.15 \\
\hline 203406_at & MFAP1 & Microfibrillar-associated protein 1 & 0.167 & -1.14 \\
\hline 224374_s_at & EMILIN2 & Elastin microfibril interfacer 2 & 0.569 & -1.12 \\
\hline 213123_at & MFAP3 & Microfibrillar-associated protein 3 & 0.257 & -1.10 \\
\hline 204534_at & $\mathrm{VTN}^{\mathrm{c}}$ & Vitronectin & 0.197 & -1.09 \\
\hline 201893_x_at & DCN & Decorin & 0.532 & -1.09 \\
\hline 213905_x_at & BGN & Biglycan & 0.681 & -1.07 \\
\hline 202994_s_at & FBLN1 & Fibulin 1 & 0.802 & -1.07 \\
\hline 203886_s_at & FBLN2 & Fibulin 2 & 0.879 & -1.05 \\
\hline 228253_at & LOXL3 $^{\mathrm{c}}$ & Lysyl oxidase-like 3 & 0.728 & -1.03 \\
\hline 215717_s_at & $\mathrm{FBN} 2^{\mathrm{c}}$ & Fibrillin 2 & 0.943 & 1.00 \\
\hline 240117_at & $\mathrm{FBN}^{\mathrm{c}}$ & Fibrillin 3 & 0.917 & 1.00 \\
\hline 202998_s_at & LOXL2 & Lysyl oxidase-like 2 & 0.969 & 1.00 \\
\hline 221731_x_at & VCAN & Versican & 0.975 & 1.01 \\
\hline 204163_at & EMILIN1 $^{\mathrm{c}}$ & Elastin microfibril interfacer 1 & 0.895 & 1.01 \\
\hline 221152_at & COL8A $1^{\mathrm{c}}$ & Collagen, type VIII, alpha 1 & 0.798 & 1.02 \\
\hline 204345_at & COL16A1 & Collagen, type XVI, alpha 1 & 0.666 & 1.05 \\
\hline 202766_s_at & FBN1 & Fibrillin 1 & 0.758 & 1.11 \\
\hline 203088_at & FBLN5 & Fibulin 5 & 0.573 & 1.14 \\
\hline 212670_at & $\mathrm{ELN}^{\mathrm{c}}$ & Elastin & 0.431 & 1.14 \\
\hline 213765_at & MAGP2 & Microfibril-associated glycoprotein 2 & 0.515 & 1.39 \\
\hline 223690_at & LTBP2 & Latent transforming growth factor $\beta$ binding protein 2 & 0.041 & 1.58 \\
\hline 215446_s_at & LOX & Lysyl oxidase & 0.186 & 1.62 \\
\hline
\end{tabular}

Affymetrix ${ }^{\circledR}$ U133A oligonucleotide array was used to identify changes in gene expression for commonly-associated components of the elastic fibre network in young and aged skin biopsies

${ }^{a}$ Genes in bold were validated by $\mathrm{qPCR}$

b Fold change (aged/young)

c Gene expression level $<50$

subsequent $\mathrm{qPCR}$ analysis demonstrated that there was no differential expression of LTBP3 between young and aged individuals (fold change \pm 1.07 , $P=0.19$; Fig. 2a). This finding was consistent with the results of an alternative LTBP3 probe set on the microarray chip (fold change \pm 1.01 , Affy ID: 227308_x_at). To further confirm this finding, the distribution of LTBP3 was examined at the protein level. LTBP3 staining was evident throughout all layers of the epidermis but at a lower intensity in the dermis of both young and aged skin (Fig. 2b, c). The level of epidermal staining was quantified by measuring fluorescence pixel intensity using ImageJ software. In agreement with the qPCR data, LTBP3 immunostaining was observed at a comparable intensity in both young and aged epidermis $(P=0.173$; Fig. $2 \mathrm{~d})$. Therefore, we conclude that LTBP3 expression and tissue distribution is not significantly altered with advancing age. 


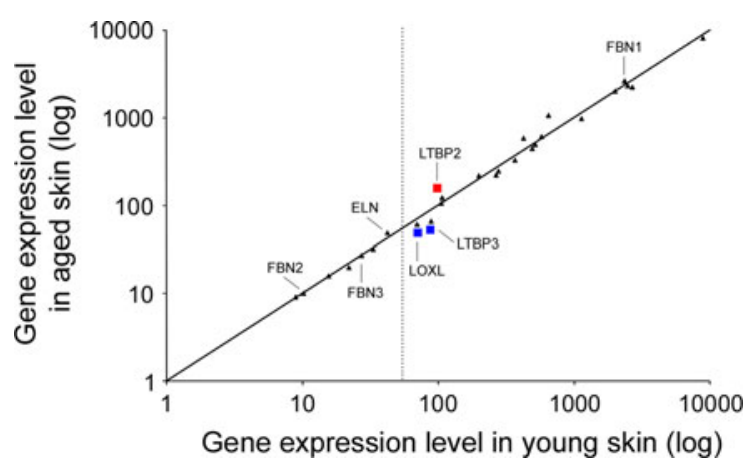

Fig. 1 Graphical representation of microarray gene expression data for elastic fibre components in young versus aged individuals. Data points situated on or close to the solid line of equivalence represent genes unchanged with age (black triangles). LTBP2 is significantly $(P=0.041)$ up-regulated with age and is positioned above the line of equivalence (red square). LTBP3 and LOXL1 are both significantly $(P=0.025$ and $P=0.008$, respectively) down-regulated with age and are positioned below the line of equivalence (blue squares). Data points located to the left of the dotted line represent gene expression values that are below the level of detection $(<50)$. Data represents mean gene expression level for $n=3$ young and aged samples

LOXL1 is up-regulated in the epidermis of intrinsically aged skin

According to microarray analysis LOXL1 expression was also down-regulated with age. However, as with the analysis of LTBP3 expression, qPCR investigations demonstrated no differential expression of LOXL1 with age (fold change +1.33 , $P=0.49$; Fig. 3a). However, whilst at the protein level, LOXL1 was localised predominantly to the basal layer of the epidermis in young skin (Fig. 3b, c), in intrinsically aged skin, LOXL1 was located in both the basal and suprabasal layers of the epidermis. In order to analyse the distribution of LOXL1 fluorescence staining present in the epidermis, line profiles extending from the DEJ to the outer surface of the skin, were generated using ImageJ software (see arrow in Fig. 3b). The fluorescence intensity of LOXL1 in the epidermis was calculated using area under the curve (AUC) analysis. Contrary to the qPCR data, aged epidermis exhibited a significant increase in the distribution of LOXL1 compared to young epidermis $(P=0.004$, Fig. 3d).
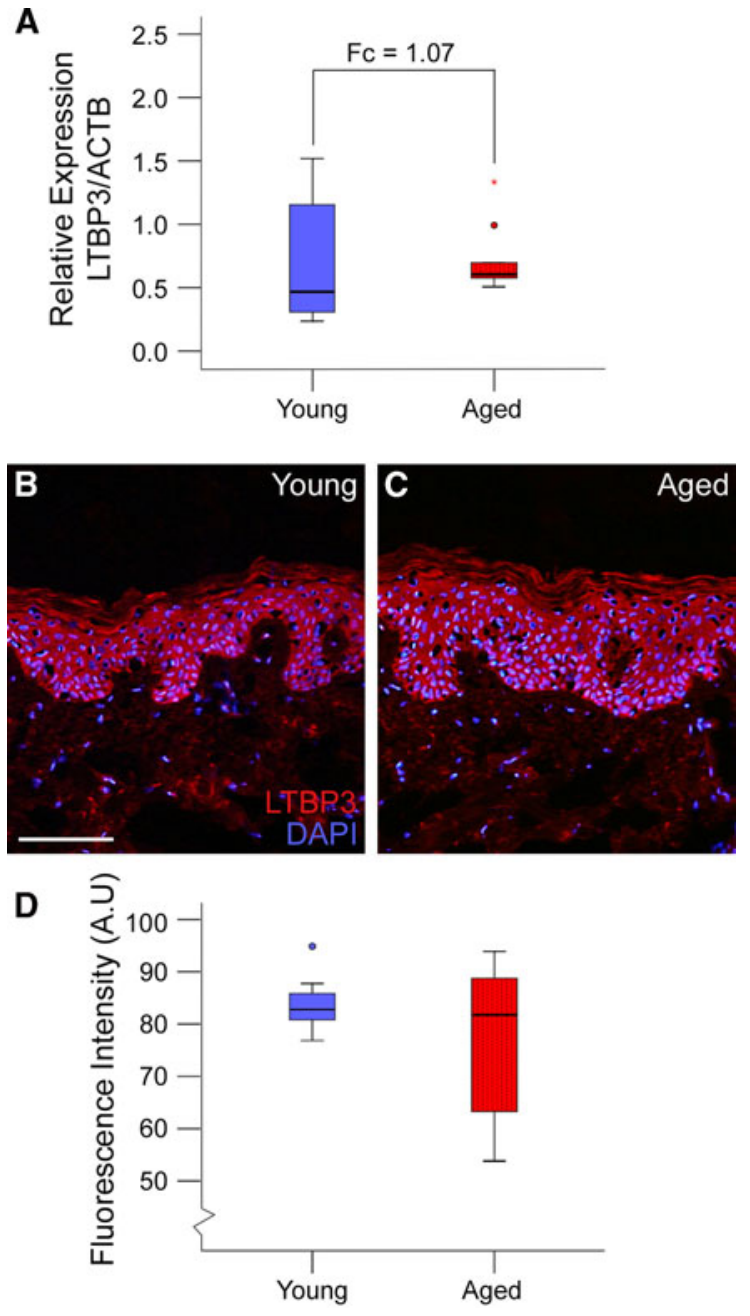

Fig. 2 qPCR was used to validate the array-determined gene expression change for LTBP3 (a). Data is represented as relative expression for LTBP3 normalised to the housekeeping gene ACTB. LTBP3 gene expression is unchanged with age, which is not consistent with the 1.67 fold down-regulation expected from the microarray (see Table 1). However, this finding was in agreement with a second probe set for LTBP3. Immunofluorescence staining of LTBP3 identifies expression throughout all layers of the epidermis in both young (b) and aged (c) skin. Epidermal fluorescence intensity of LTBP3 is unchanged with age $(P=0.173)(\mathbf{d})$. Scale bar: $100 \mu \mathrm{m}$. Data are presented as mean \pm standard error of the mean; $n=9$ for young and aged samples

LTBP2 expression is up-regulated and the protein accumulates at the DEJ in intrinsically aged skin

Microarray data suggested that LTBP2 expression was up-regulated with age and this was supported by 

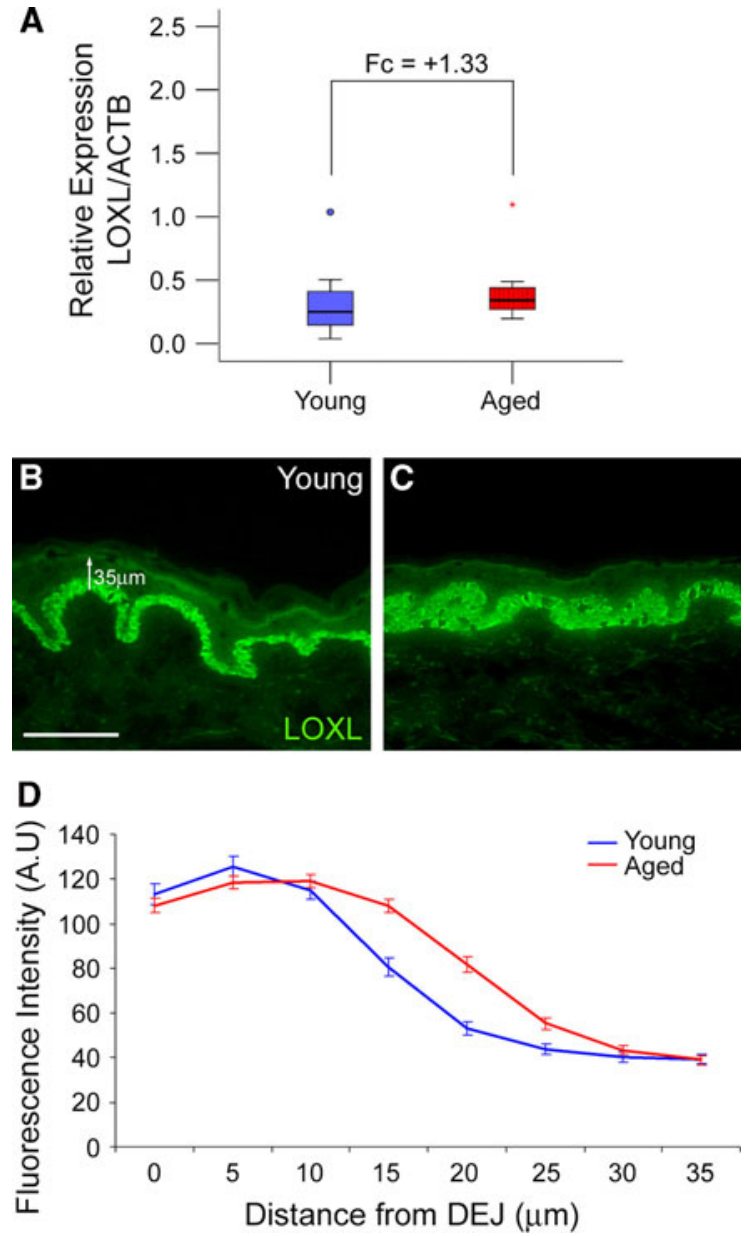

Fig. 3 qPCR was used to validate the array-determined gene expression change for LOXL1 (a). Data is represented as relative expression for LOXL1 normalised to the housekeeping gene ACTB. LOXL1 qPCR data was inconsistent with the microarray study as LOXL1 mRNA expression was unchanged with age $(P=0.49)$. Immunofluorescence staining identifies LOXL1 expression specifically in the epidermal basal layer in young (b) and aged (c) skin. Non-specific staining was identified in the stratum corneum. The distribution of epidermal LOXL1 was analysed by plotting line profiles of fluorescence intensity from the DEJ towards the outer surface of the skin (see arrow in (b)) for both young and aged skin (d). Epidermal LOXL1 distribution is significantly increased in aged skin compared to young skin $(P=0.004)$. Scale bar: $100 \mu \mathrm{m}$. Data are presented as mean \pm standard error of the mean; $n=9$ for young and aged samples

qPCR analysis (fold change +1.56, $P<0.05$; Fig. 4a). In order to visualise the distribution of LTBP2 in skin, two commercially-available antibodies were used. The first, a polyclonal antibody was raised against the amino acid sequence at 241-376 of the LTBP2 protein. Using this antibody, the age-dependent tissue distribution of LTBP2 resembled the distribution of LOXL1. Specifically, LTBP2 was localised to the epidermal basal layer in young skin (Fig. 4b) and to the basal and suprabasal layers in aged skin (Fig. 4c). No dermal staining was noted in either the young or aged samples. The distribution of LTBP2 fluorescence staining present in the epidermis was analysed using line profiles extending from the DEJ to the outer surface of the skin over a distance of $50 \mu \mathrm{m}$ (see arrow in Fig. 4b). The relative fluorescence intensity of LTBP2 staining in the epidermis was calculated using AUC analysis. In aged epidermis there was a significant increase in the distribution of LTBP2 staining compared to young skin $(P<0.001$; Fig. 4 d), which was consistent with the qPCR data.

The second antibody used to examine LTBP2 abundance and distribution was a monoclonal targeting the amino acid sequence at 1709-1819 of the protein. In both young and aged samples, no epidermal LTBP2 staining was noted. However, considerable LTBP2 staining was found throughout the dermis in the young samples (Fig. 4e). In aged dermis, LTBP2 was localised predominantly at the DEJ and was sparsely distributed throughout the dermis (Fig. 4f). Line profiles extending from the DEJ into the deeper dermis were used to analyse LTBP2 distribution over a distance of $100 \mu \mathrm{m}$ (see arrow in Fig. 4e). In young skin, LTBP2 was organised into an array which closely resembles the previously reported dermal distribution of fibrillin-1 (Fig. 4g) (Watson et al. 1999). However, in aged skin LTBP2 was distributed predominantly in the first $20 \mu \mathrm{m}$ of dermis from the DEJ $(P=0.05)$ (Fig. $4 \mathrm{~g})$. In the remaining $80 \mu \mathrm{m}$ of the dermis analysed, LTBP2 was distributed in a microfibrillar array but the overall distribution was reduced compared to young dermis $(P<0.006)$.

\section{Discussion}

This study used a combined microarray, qPCR and immunohistochemical approach to quantify the expression and the distribution of elastic fibre components in intrinsically aged skin (see Table 2 for a summary of the experimental findings by technique for LTBP2, LTBP3 and LOXL). The majority of elastic fibre-associated components were found to be 
A
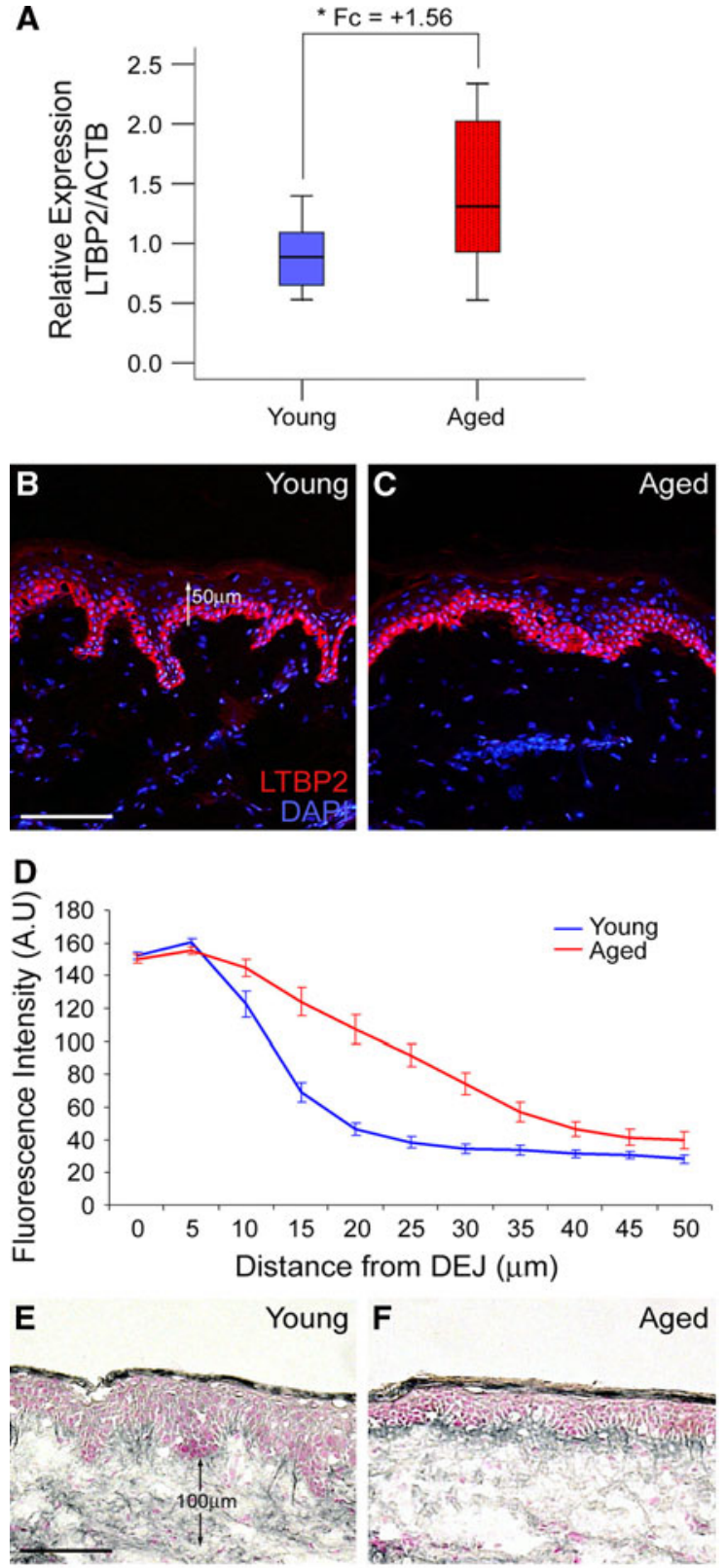

Aged

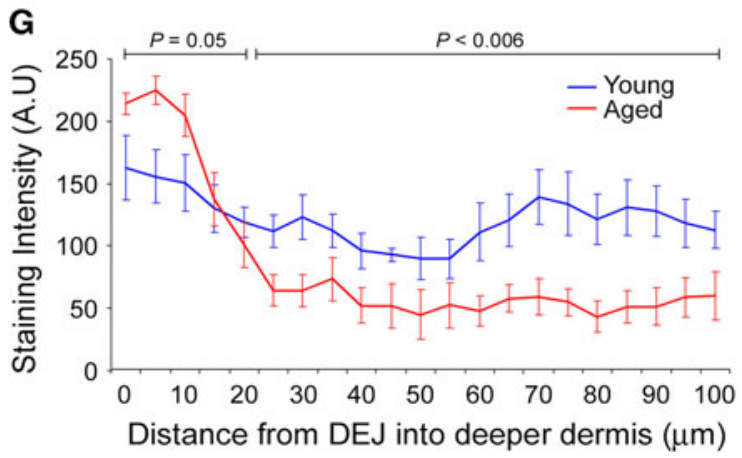

4 Fig. 4 qPCR was used to validate the array-determined gene expression change for LTBP2 (a). Data is represented as relative expression for LTBP2 normalised to the housekeeping gene ACTB. qPCR confirmed a significant 1.6 fold up-regulation of LTBP2 in aged skin $(P<0.05)$, which was consistent with the microarray data. Immuno-localisation of LTBP2 expression was determined using two different commercially available antibodies. Using an antibody targeting the amino acid sequence at 241-376 of the LTBP2 protein, immunofluorescence staining identifies expression specifically in the epidermal basal layer in young skin (b). In aged epidermis, LTBP2 is expressed in both the basal and suprabasal layers of the epidermis (c). No dermal staining is noted in either young or aged samples. Distribution of epidermal LTBP2 was analysed by plotting line profiles of fluorescence intensity from the DEJ towards the outer surface of the skin (see arrow in (b)) for both young and aged skin (d). Epidermal LTBP2 distribution is significantly increased in aged skin compared to young skin $(P<0.001)$. Immunohistochemical staining was performed using a second antibody targeting the amino acid sequence at $1709-1819$ of the LTBP2 protein. LTBP2 expression is distributed consistently throughout all layers of the dermis in young skin (e) whereas in aged dermis, LTBP2 is localised predominantly at the DEJ and is sparsely distributed throughout the dermis (f). The distribution of dermal LTBP2 was analysed by plotting line profiles of pixel intensity from the DEJ towards the deeper layers of the dermis (see arrow in (e)) for both young and aged skin (f). LTBP2 was found to be predominantly distributed in the first $20 \mu \mathrm{m}$ of dermis in aged skin $(P=0.05)$. However, the distribution of LTBP2 staining within the remaining dermis was significantly reduced in aged skin $(P<0.006)(\mathbf{g})$. Scale bar: $100 \mu \mathrm{m}$. Data are presented as mean \pm standard error of the mean; $n=9$ for young and aged samples

unchanged with age. However, given the subtle changes observed clinically and histologically during intrinsic ageing, this was not entirely unexpected. Of the three genes which did show differential expression by microarray analysis, only the modulation of LTBP2 expression was validated by qPCR. These observations support the view that whilst microarray analysis is a rapid means of identifying potentially important changes in gene expression there remains a strong requirement for robust validation of any findings using more sensitive techniques, such as qPCR or northern blot (White and Salamonsen 2005).

Such mRNA expression data gives a partial indication of the cellular phenotype at a given moment in time. However, in order to more fully characterise age-related changes it is necessary to assess the abundance and distribution of ECM proteins which in general have half lives that are measured in years (Shapiro et al. 1991; Davis 1993). Immunostaining determined that LTBP3 was equally 
Table 2 Summary of findings for components identified in this study

\begin{tabular}{lllll}
\hline & LTBP2 & LTBP3 & LTBP3 & LOXL1 \\
& Affy ID: 223690_at & Affy ID: 219922_s_at & Affy ID: 227308_x_at & Affy ID: 203570_at \\
\hline Affymetrix $^{\circledR}$ & $\uparrow$ & $\downarrow$ & $\leftrightarrow$ & $\downarrow$ \\
qPCR & $\uparrow$ & $\leftrightarrow$ & $\leftrightarrow$ & $\uparrow$ \\
Immunostaining & $\uparrow$ (epidermis) $\downarrow$ (dermis) & $\leftrightarrow$ & $\leftrightarrow$ & $\uparrow$ \\
\hline
\end{tabular}

A summary of the findings, by technique, for each elastic fibre component analysed in this study

abundant in the epidermis of both young and aged individuals and we therefore conclude that LTBP3 is unaffected by intrinsic ageing.

Similarly, LOXL1 protein was also localised to the epidermis in both young and aged skin. This observation is consistent with previous reports of LOXL1 expression in human and murine skin (Noblesse et al. 2004; Liu et al. 2004). LOXL1 is a key component of elastic fibres with LOXL1 knock-out mice exhibiting increased skin laxity (Liu et al. 2004). Although the primary role of LOXL1 is to cross-link elastin, it has also been shown to be an essential element of the scaffold onto which elastin is deposited (Liu et al. 2004). It is uncertain why LOXL1 should be predominately located in the epidermis rather than adjacent to the elastic fibre system in the dermis. However, previous in situ hybridisation studies have localised abundant expression of the key elastic fibre component fibrillin-1 (Watson et al. 1999) and of LOXL1 itself (Noblesse et al. 2004) to the epidermis. Such tissue distributions suggest that epidermal keratinocytes have the potential to make a major contribution to the deposition of the dermal elastic fibre system microfibrillar arrays in normal skin (Watson et al. 1999).

The protein distribution of LTBP2 was markedly altered in the epidermis and dermis of aged skin however; in order to detect these changes, two different antibodies were utilised. The differential distribution of these two antibodies suggests that alterations in the tertiary structure of LTBP2 may occur with age and that such changes may be dependent on skin anatomy. Thus, LTBP2 has the epitope recognisable by the first antibody but not the second in the epidermis and vice versa in the papillary and reticular dermis. The function of LTBP2 is currently unknown; however, it is critical during embryogenesis as LTBP2-deficient mice do not survive past the implantation stage of development
(Shipley et al. 2000). LTBP2 and LTBP3 are two of the four currently-identified LTBP family members (LTBP1-4) (Kanzaki et al. 1990; Moren et al. 1994; Yin et al. 1995; Giltay et al. 1997; Saharinen et al. 1998). Most LTBPs are able to sequester latent TGF $\beta$, with the exception of LTBP2 (Saharinen and Keski-Oja 2000). LTBP2 is however, known to associate with developing microfibrils and is considered to perform a structural role within elastic fibres (Gibson et al. 1995). More specifically, LTBP2 has been shown to bind directly via its carboxy-terminal region to the amino-terminal region of fibrillin-1 (Hirani et al. 2007).

Interestingly, LOXL1 and LTBP2 are both binding partners of a third elastic fibre component, fibulin-5 (Liu et al. 2004; Hirai et al. 2007). Fibulin-5 is located at the microfibril/elastin interface and localises specifically to sites of elastogenesis (Nakamura et al. 2002; Reinhardt et al. 1996). In a similar manner as seen for LOXL1-deficient mice, fibulin-5-deficient mice have loose skin due to disorganisation of the dermal elastic fibres (Nakamura et al. 2002). In these mice, loss of fibulin-5 protein abolishes the fibrillar staining pattern of LOXL1 in the deep dermis, but not vice versa (Liu et al. 2004). The interaction between LTBP2 and fibulin-5 promotes the deposition of fibulin-5 onto fibrillin-rich microfibrils (Hirai et al. 2007) and it is hypothesised that LTBP2 drives the correct assembly of elastic fibres by acting as a molecular switch to determine which microfibrils to deposit fibulin-5 onto (Hirai et al. 2007).

Expression of fibulin-5 during intrinsic skin ageing has previously been characterised (Kadoya et al. 2005). In young photoprotected skin, fibulin-5 is localised to the elastic fibres throughout the dermis, however in aged skin this pattern of fibulin-5 staining is completely abolished (Kadoya et al. 2005). The loss of fibulin-5 staining during intrinsic skin ageing was confirmed for the aged samples used in this study 


\section{Young skin}

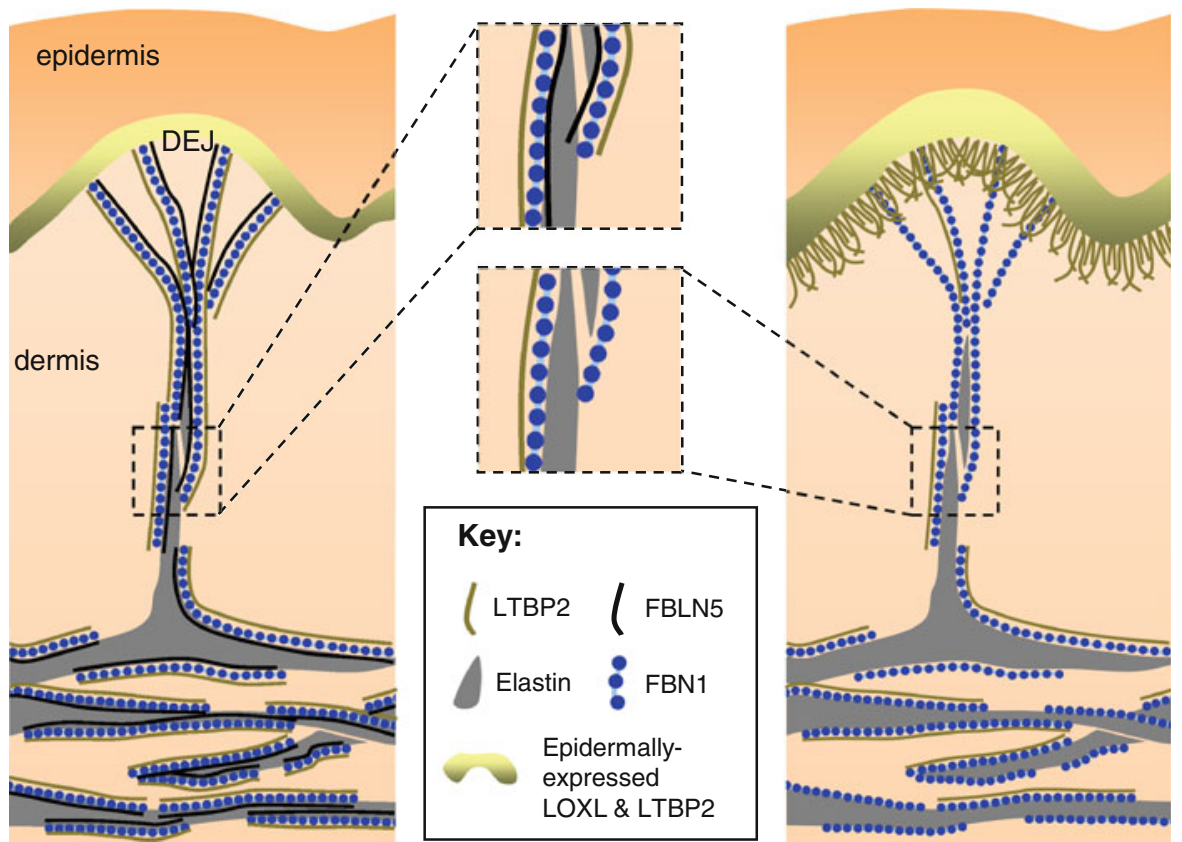

Fig. 5 A schematic representation of elastic fibre component distribution in young and intrinsically aged skin. In young skin the elastic fibres of the dermis form a microfibrillar arrangement. The fibres consist predominantly of an elastin core and an outer mantle of fibrillin-rich microfibrils. In addition, LTBP2 is bound to the microfibrils and FBLN5 to the microfibril-elastin interface. In intrinsically aged skin, the microfibrillar arrangement of fibrillin-1 and elastin remains. However, FBLN5 is completely abolished from the dermis and LTBP2 accumulates at the DEJ. In addition, the distribution of LOXL and LTBP2 increases in aged epidermis (data not shown). Accumulation of LTBP2 and LOXL1 at or around the DEJ suggests that abolition of fibulin-5 in the dermis causes profound perturbation to the correct deposition of these components in aged skin. If LTBP2 and LOXL1 both require fibulin5 as the scaffold via which they exert their function, it is possible that in its absence these components would accumulate elsewhere in the skin (Fig. 5). Furthermore, the continual transcription and translation of LOXL1 and LTBP2 in aged skin may represent an attempt by the basal keratinocytes to compensate for the loss of fibulin-5.

In conclusion, the microarray results indicated that the majority of elastic fibre-associated components were unchanged with age. This supports the hypothesis that elastic fibre proteins are long-lived biomolecules and that ageing is due to the remodelling of existing proteins-potentially by acellular mechanisms (Sherratt et al. 2010). Given that intrinsic ageing of the skin is a slow and subtle process it is surprising that significant alteration in expression for LTBP2 and LOXL1 have been identified. This indicates that these components in particular may impact greatly on the organisation of elastic fibres in intrinsically aged skin. We hypothesise that changes in LTBP2 and LOXL1 protein distribution during intrinsic skin ageing may be influenced by the loss of the fibulin-5 scaffold onto which these proteins bind. This may have consequences for both the mechanical properties and homeostasis of the elastic fibres and could contribute to the clinical signs of ageing such as skin laxity and wrinkling.

Acknowledgments We are grateful to Jean Bastrilles, Gill Aarons and June Bowden for recruitment of volunteers and the University of Manchester Genomic Technologies Core Facility (formerly Microarray Core Facility). This work was supported by a programme grant from Alliance Boots, Nottingham, UK and by a Senior Age UK Fellowship awarded to MJS. CEMG is supported in part by the NIHR Manchester Biomedical Research Centre. 


\section{References}

Abramoff MD, Magelhaes PJ, Ram SJ (2004) Image processing with image. J. Biophotonics International 11:36-42

Ashworth JL, Murphy G, Rock MJ, Sherratt MJ, Shapiro SD, Shuttleworth CA, Kielty CM (1999) Fibrillin degradation by matrix metalloproteinases: implications for connective tissue remodelling. Biochem J 340(Pt 1):171-181

Baldi P, Long AD (2001) A Bayesian framework for the analysis of microarray expression data: regularized t-test and statistical inferences of gene changes. Bioinformatics 17(6):509-519

Braverman IM, Fonferko E (1982) Studies in cutaneous aging: I. The elastic fiber network. J Invest Dermatol 78(5): 434-443

Cotta-Pereira G, Guerra Rodrigo F, Bittencourt-Sampaio S (1976) Oxytalan, elaunin, and elastic fibers in the human skin. J Invest Dermatol 66(3):143-148

Davis EC (1993) Stability of elastin in the developing mouse aorta: a quantitative radioautographic study. Histochemistry $100(1): 17-26$

Ernster VL, Grady D, Miike R, Black D, Selby J, Kerlikowske K (1995) Facial wrinkling in men and women, by smoking status. Am J Public Health 85(1):78-82

Escoffier C, de Rigal J, Rochefort A, Vasselet R, Leveque JL, Agache PG (1989) Age-related mechanical properties of human skin: an in vivo study. J Invest Dermatol 93(3): 353-357

Fisher GJ, Datta SC, Talwar HS, Wang ZQ, Varani J, Kang S, Voorhees JJ (1996) Molecular basis of sun-induced premature skin ageing and retinoid antagonism. Nature 379(6563):335-339. doi:10.1038/379335a0

Giangreco A, Goldie SJ, Failla V, Saintigny G, Watt FM (2010) Human skin aging is associated with reduced expression of the stem cell markers betal integrin and MCSP. J Invest Dermatol 130(2):604-608. doi:10.1038/ jid.2009.297

Gibson MA, Hatzinikolas G, Davis EC, Baker E, Sutherland GR, Mecham RP (1995) Bovine latent transforming growth factor beta 1-binding protein 2: molecular cloning, identification of tissue isoforms, and immunolocalization to elastin-associated microfibrils. Mol Cell Biol 15(12): 6932-6942

Giltay R, Kostka G, Timpl R (1997) Sequence and expression of a novel member (LTBP-4) of the family of latent transforming growth factor-beta binding proteins. FEBS Lett 411(2-3):164-168

Hirai M, Horiguchi M, Ohbayashi T, Kita T, Chien KR, Nakamura T (2007) Latent TGF-beta-binding protein 2 binds to DANCE/fibulin-5 and regulates elastic fiber assembly. EMBO J 26(14):3283-3295. doi:10.1038/sj.emboj.7601 768

Hirani R, Hanssen E, Gibson MA (2007) LTBP-2 specifically interacts with the amino-terminal region of fibrillin-1 and competes with LTBP-1 for binding to this microfibrillar protein. Matrix Biol 26(4):213-223. doi:10.1016/j.matbio. 2006.12.006

Kadoya K, Sasaki T, Kostka G, Timpl R, Matsuzaki K, Kumagai N, Sakai LY, Nishiyama T, Amano S (2005) Fibulin-5 deposition in human skin: decrease with ageing and ultraviolet B exposure and increase in solar elastosis. Br J Dermatol 153(3):607-612. doi:10.1111/j.1365-2133. 2005.06716.x

Kadunce DP, Burr R, Gress R, Kanner R, Lyon JL, Zone JJ (1991) Cigarette smoking: risk factor for premature facial wrinkling. Ann Intern Med 114(10):840-844

Kafi R, Kwak HS, Schumacher WE, Cho S, Hanft VN, Hamilton TA, King AL, Neal JD, Varani J, Fisher GJ, Voorhees JJ, Kang S (2007) Improvement of naturally aged skin with vitamin A (retinol). Arch Dermatol 143(5): 606-612. doi:10.1001/archderm.143.5.606

Kanzaki T, Olofsson A, Moren A, Wernstedt C, Hellman U, Miyazono K, Claesson-Welsh L, Heldin CH (1990) TGFbeta 1 binding protein: a component of the large latent complex of TGF-beta 1 with multiple repeat sequences. Cell 61(6):1051-1061

Kielty CM, Woolley DE, Whittaker SP, Shuttleworth CA (1994) Catabolism of intact fibrillin microfibrils by neutrophil elastase, chymotrypsin and trypsin. FEBS Lett 351(1):85-89

Kielty CM, Sherratt MJ, Shuttleworth CA (2002) Elastic fibres. J Cell Sci 115(Pt 14):2817-2828

Langton AK, Sherratt MJ, Griffiths CEM, Watson REB (2010) A new wrinkle on old skin: the role of elastic fibres in skin ageing. Int J Cosmet Sci. doi:10.1111/j.1468-2494.2010. 00574.x

Li C, Wong WH (2001) Model-based analysis of oligonucleotide arrays: expression index computation and outlier detection. Proc Natl Acad Sci USA 98(1):31-36. doi: 10.1073/pnas.011404098

Liu X, Zhao Y, Gao J, Pawlyk B, Starcher B, Spencer JA, Yanagisawa H, Zuo J, Li T (2004) Elastic fiber homeostasis requires lysyl oxidase-like 1 protein. Nat Genet 36(2):178-182. doi:10.1038/ng 1297

Mecham RP, Davis EC (1994) Elastic fiber structure and assembly. In: Yurchenco PD, Birk DE, Mecham RP (eds) Extracellular matrix assembly and structure. Academic Press, New York, pp 281-314

Mithieux SM, Weiss AS (2005) Elastin. Adv Protein Chem 70:437-461. doi:10.1016/S0065-3233(05)70013-9

Montagna W, Carlisle K (1979) Structural changes in aging human skin. J Invest Dermatol 73(1):47-53

Moren A, Olofsson A, Stenman G, Sahlin P, Kanzaki T, Claesson-Welsh L, ten Dijke P, Miyazono K, Heldin CH (1994) Identification and characterization of LTBP-2, a novel latent transforming growth factor-beta-binding protein. J Biol Chem 269(51):32469-32478

Nakamura T, Lozano PR, Ikeda Y, Iwanaga Y, Hinek A, Minamisawa S, Cheng CF, Kobuke K, Dalton N, Takada Y, Tashiro K, Ross J Jr, Honjo T, Chien KR (2002) Fibulin$5 / \mathrm{DANCE}$ is essential for elastogenesis in vivo. Nature 415(6868):171-175. doi:10.1038/415171a

Noblesse E, Cenizo V, Bouez C, Borel A, Gleyzal C, Peyrol S, Jacob MP, Sommer P, Damour O (2004) Lysyl oxidaselike and lysyl oxidase are present in the dermis and epidermis of a skin equivalent and in human skin and are associated to elastic fibers. J Invest Dermatol 122(3): 621-630. doi:10.1111/j.0022-202X.2004.22330.x

Saharinen J, Keski-Oja J (2000) Specific sequence motif of 8 -Cys repeats of TGF-beta binding proteins, LTBPs, 
creates a hydrophobic interaction surface for binding of small latent TGF-beta. Mol Biol Cell 11(8):2691-2704

Saharinen J, Taipale J, Monni O, Keski-Oja J (1998) Identification and characterization of a new latent transforming growth factor-beta-binding protein, LTBP-4. J Biol Chem 273(29):18459-18469

Shapiro SD, Endicott SK, Province MA, Pierce JA, Campbell EJ (1991) Marked longevity of human lung parenchymal elastic fibers deduced from prevalence of D-aspartate and nuclear weapons-related radiocarbon. J Clin Invest 87(5):1828-1834. doi:10.1172/JCI115204

Sherratt MJ (2009) Tissue elasticity and the ageing elastic fibre. Age (Dordr) 31(4):305-325. doi:10.1007/s11357009-9103-6

Sherratt MJ, Bayley CP, Reilly SM, Gibbs NK, Griffiths CE, Watson RE (2010) Low-dose ultraviolet radiation selectively degrades chromophore-rich extracellular matrix components. J Pathol 222(1):32-40. doi:10.1002/path.2730

Shipley JM, Mecham RP, Maus E, Bonadio J, Rosenbloom J, McCarthy RT, Baumann ML, Frankfater C, Segade F, Shapiro SD (2000) Developmental expression of latent transforming growth factor beta binding protein 2 and its requirement early in mouse development. Mol Cell Biol 20(13):4879-4887

Storey JD, Tibshirani R (2003) Statistical significance for genomewide studies. Proc Natl Acad Sci USA 100(16): 9440-9445. doi:10.1073/pnas.1530509100
Uitto J (1979) Biochemistry of the elastic fibers in normal connective tissues and its alterations in diseases. J Invest Dermatol 72(1):1-10

Watson REB, Griffiths CEM, Craven NM, Shuttleworth CA, Kielty CM (1999) Fibrillin-rich microfibrils are reduced in photoaged skin. Distribution at the dermal-epidermal junction. J Invest Dermatol 112(5):782-787. doi:10.1046/ j.1523-1747.1999.00562.x

Watson REB, Craven NM, Kang S, Jones CJ, Kielty CM, Griffiths CEM (2001) A short-term screening protocol, using fibrillin-1 as a reporter molecule, for photoaging repair agents. J Invest Dermatol 116(5):672-678. doi: 10.1046/j.1523-1747.2001.01322.x

White CA, Salamonsen LA (2005) A guide to issues in microarray analysis: application to endometrial biology. Reproduction 130(1):1-13. doi:10.1530/rep.1.00685

Wu Z, Irizarry RA, Gentleman R, Murillo FM, Spencer F (2004) A model-based background adjustment for oligonucleotide expression arrays. Working Papers, Department of Biostatistics, John Hopkins University

Yin W, Smiley E, Germiller J, Mecham RP, Florer JB, Wenstrup RJ, Bonadio J (1995) Isolation of a novel latent transforming growth factor-beta binding protein gene (LTBP-3). J Biol Chem 270(17):10147-10160 